\title{
Techniques for Contextual Equivalence in Higher-Order, Typed Languages
}

\author{
Andrew Pitts \\ University of Cambridge
}

\begin{abstract}
Two phrases in a programming language are said to be contextually equivalent if, roughly speaking, they are interchangeable in any complete program without affecting the observable behaviour of the program. I will discuss precise formalisations of this fundamental notion of semantic equivalence for the case of higher-order, typed (HOT) languages, such as ML and Haskell. How does the structure of a type affect properties of contextual equivalence of expressions of that type? It can be very difficult to answer this question when working directly from the definition of contextual equivalence-mainly because HOT programs can make use of their constituent sub-expressions in dynamically complicated ways. This talk will survey some of the semantic techniques (both denotational and operational) that have been devised for proving properties of HOT contextual equivalence.
\end{abstract}

